\title{
Modelling the depletion length induced by modulation instability in distributed optical fibre sensors
}

\author{
Mehdi Alem*, Marcelo A. Soto, and Luc Thévenaz \\ EPFL Swiss Federal Institute of Technology, Group for Fiber Optics (GFO), SCI-STI-LT, Station 11, \\ CH-1015 Lausanne, Switzerland \\ *E-mail: mehdi.alem@epfl.ch
}

\begin{abstract}
An analytical model for the depletion length of modulation instability in single-mode optical fibres is proposed. The model gives the possibility to determine the maximum sensing distance that distributed optical fibre sensors can reach before being limited by the pump depletion induced by modulation instability. The important role of the noise level in the evolution of both modulation instability and the respective power depletion is clarified. The model gives a closedform expression helpful for a predictive design and is validated comparing the analytical results obtained by the model with measurements in a $25 \mathrm{~km}$ long Brillouin fibre sensor.
\end{abstract}

Keywords: Modulation instability, optical fibre sensor, distributed fibre sensor, power depletion

\section{INTRODUCTION}

Distributed optical fibre sensors are widely used for temperature and strain monitoring due to their unique advantages such as long continuous sensing range and high spatial resolution. For long distance measurements, high pump power has to be launched into the sensing fibre to compensate fibre attenuation and to reach an adequate signal-to-noise ratio (SNR) at the far end of the fibre. However, increasing the pump power above a critical level gives rise to large nonlinear effects, such as modulation instability (MI) and stimulated Raman scattering (SRS), which deplete the pump power and degrade the performance of the sensing system ${ }^{1}$. Compared with SRS, MI has a lower threshold and thus is the main limitation for increasing the pump power ${ }^{1}$. Modulation instability can be avoided using optical fibres with normal dispersion; however, standard single-mode fibres (SMF) are preferably used in fibre sensors for performance and economic reasons, so that their anomalous dispersion makes them necessarily subject to $\mathrm{MI}^{2}$. Modulation instability has detrimental impacts on different types of distributed fibre sensors, imposing a fundamental limit to their maximum sensing distance. For instance, in Brillouin optical time-domain reflectometry (BOTDR) ${ }^{3}$, MI contaminates the detected Brillouin signal in addition to pump depletion. This induces an upward offset in the measured Brillouin frequency, which biases the measured temperature/strain along the fibre ${ }^{3}$. On the other hand, in Brillouin optical time-domain analysis (BOTDA) fibre sensors ${ }^{4}$, the pump depletion due to MI is particularly critical since the gain of stimulated Brillouin scattering (SBS) and hence the sensor response depends directly on the pump power ${ }^{1}$. In addition, MI also induces visibility fading in phase sensitive OTDR systems 5 . While for SRS a simple and accurate model was introduced by Smith $^{6}$, matching very well experimental results ${ }^{1}$, for modulation instability the analytical models presented so far show a discrepancy with experimental measurements ${ }^{1,7}$. In this work, one step forward is taken to define a criterion and obtain a simple expression for the power threshold of MI. Two parameters, namely depletion ratio and depletion length, are introduced to analytically model the impact of MI in distributed fibre sensors. From this, a quite simple formula for depletion length is presented in a closed-form expression. The analytical model is compared with numerical solutions, Monte Carlo simulations, and experimental measurements in a standard BOTDA system.

\section{ANALYTICAL MODEL}

Modulation instability was observed in optical fibres for the first time by Tai, et al. ${ }^{8}$ It is a process in which a tiny modulation of the optical field grows exponentially as a result of an interplay between the group velocity dispersion (GVD) in anomalous regime $\left(\beta_{2}<0\right)$ and the nonlinear Kerr effect represented by the nonlinear coefficient $\gamma$. The MI gain spectrum can be obtained by applying a linear perturbation analysis to the nonlinear Schrödinger (NLS) equation ${ }^{8}$. In undepleted pump regime with input power $P_{0}$ and for a lossless fibre, the spectral distribution of the MI gain at a point $z$ along the fibre is expressed by ${ }^{7}$ :

23rd International Conference on Optical Fibre Sensors, edited by José Miguel López-Higuera,

Julian Jones, Manuel López-Amo, José Luis Santos, Proc. of SPIE Vol. 9157, 91575S

(C) 2014 SPIE · CCC code: 0277-786X/14/\$18 · doi: 10.1117/12.2058862 


$$
G_{\mathrm{MI}}(f)=1+\frac{\sinh ^{2}\left(2 \gamma P_{0} z \sqrt{\left(\frac{f}{f_{c}}\right)^{2}\left(1-\left(\frac{f}{f_{c}}\right)^{2}\right)}\right)}{2\left(\frac{f}{f_{c}}\right)^{2}\left(1-\left(\frac{f}{f_{c}}\right)^{2}\right)},
$$

where $f=v-v_{0}$ is the frequency detuning from the pump frequency $v_{0}$, and the cut-off frequency $f_{c}=\frac{1}{\pi} \sqrt{\frac{\gamma P_{0}}{\left|\beta_{2}\right|}}$ is the maximum detuning fixing the boundaries of the MI gain frequency band. For lossy fibres, although the gain formula is much more complicated ${ }^{9}$, replacing the length $z$ by the effective length $z_{\mathrm{eff}}=\left(1-e^{-\alpha z}\right) / \alpha$ and multiplying Eq. (1) by the factor $e^{-\alpha z}$ can be an acceptable approximation, where $\alpha$ is the attenuation coefficient of the fiber. The MI gain reaches its peak value $1+2 \sinh ^{2}\left(\gamma P_{0} z\right)$ at the frequencies $\pm f_{\mathrm{c}} / \sqrt{2}$. Fig. 1 depicts the MI gain spectrum in a singlemode fibre, obtained from Eq. (1) and also by simulating the NLS equation using split-step Fourier transform. The figure shows a perfect match between the analytical solution and the Monte Carlo simulation.

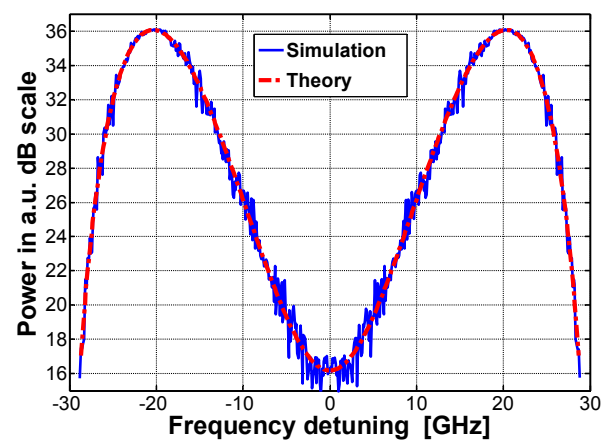

Figure 1. MI gain spectrum for a $25 \mathrm{~km} \mathrm{SMF}$ with $100 \mathrm{~mW}$ input power and typical values of $\gamma=1.8(\mathrm{kmW})^{-1}$ and $\beta_{2}=-22 \mathrm{ps}^{2} / \mathrm{km}$.

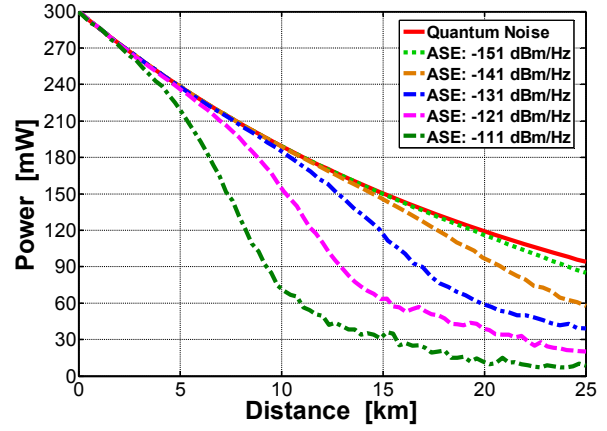

Figure 2. Depletion of pump power $\left(P_{0}=300 \mathrm{~mW}\right)$ propagating through a $25 \mathrm{~km} \mathrm{SMF}$ with $\alpha=0.2 \mathrm{~dB} / \mathrm{km}$ for different levels of noise $S_{n}$

During the evolution of the MI process along the fibre, the optical pump power is gradually transferred to two symmetric spectral sidebands, resulting in depletion of the pump power at its original frequency. The background noise present over the MI frequency band plays the role of a signal seeding the MI process; so that the occurrence of modulation instability and the amount of pump depletion depend on the background noise level propagating in the fibre. This noise mainly results from the amplified spontaneous emission (ASE) in optical amplifiers ${ }^{7}$. Fig. 2 shows the effect of pump depletion due to modulation instability as the pump power propagates along a standard SMF with attenuation coefficient $\alpha=$ $0.2 \mathrm{~dB} / \mathrm{km}$, for different levels of background noise. If $S_{n}$ denotes the power spectral density of the background noise, the total power transferred to the MI frequency bands is obtained via the following expression:

$$
P_{\mathrm{MI}}=\int_{0}^{\infty} G_{\mathrm{MI}}(v) S_{n} d v
$$

There are two options for solving the above integral: one is the numerical calculation resulting in an exact but nonclosed-form solution, and the other is the analytical solution leading to an approximate but closed-form expression for the MI power. Using the latter approach also provides a better insight into the depletion process, resulting in a useful design tool for distributed optical fibre sensors. Thus, assuming a white spectral density for the background noise, the following simple equation, relating the main parameters involved the MI process, can be obtained:

$$
2 \gamma P_{0} z_{\mathrm{eff}}=\operatorname{Ln}\left(\frac{P_{\mathrm{MI}}}{f_{c} S_{n}}\right)+\alpha z
$$

In order to relate the MI power to the pump power, the depletion ratio $R_{\mathrm{D}}$ is introduced as the ratio of the depleted power to the undepleted ideal case, as follows:

$$
R_{\mathrm{D}}=\frac{P_{0} e^{-\alpha z}-P(z)}{P_{0} e^{-\alpha z}}=1-\frac{P(z)}{P_{0} e^{-\alpha z}}
$$

where $P(z)$ is the pump power at position $z$ considering pump depletion. Since the MI power indicates the amount of depleted pump power, $P_{\mathrm{MI}}$ can be expressed as $R_{\mathrm{D}} P_{0} e^{-\alpha z}$. Therefore, substituting $P_{\mathrm{MI}}$ and $f_{c}$ in Eq. (3), the following 
analytic expression for the depletion length due to the modulation instability is obtained in terms of the input pump power, depletion ratio, background noise level and fibre parameters:

$$
2 \gamma P_{0} Z_{\text {eff }}=\operatorname{Ln}\left(\frac{\pi R_{\mathrm{D}}}{S_{n}} \sqrt{\frac{\left|\beta_{2}\right|}{\gamma}} \sqrt{P_{0}}\right) .
$$

It is worth mentioning that, in order to obtain the power threshold for modulation instability, it is essential to solve the above expression as a transcendental equation for the input pump power $P_{0}$. However, the depletion length $L_{\mathrm{D}}$ can be obtained calculating $z_{\text {eff }}$ directly from Eq. (5) and then substituting $z_{\text {eff }}$ into $L_{\mathrm{D}}=-\operatorname{Ln}\left(1-\alpha z_{\text {eff }}\right) / \alpha$.

\section{EXPERIMENTAL RESULTS}

To verify the proposed analytical model, a standard BOTDA system, sketched in Fig. 3, is used to measure the Brillouin gain versus the fibre length. Since the Brillouin gain at each fibre location is proportional to the local pump power, by acquiring the Brillouin trace along the fibre at the peak gain frequency, the evolution of pump power versus the fibre length can be determined. A laser diode (LD) at $1551.1 \mathrm{~nm}$ is coupled into the pump and probe branches by a 50:50 coupler. A semiconductor optical amplifier (SOA) is used to generate pump pulses with high extinction ratio. The pulses are amplified by an erbium-doped fibre amplifier (EDFA) and attenuated by a variable optical attenuator (VOA) to precisely adjust and vary the pump peak power. The power calibration unit, shown in the dashed box, is used to monitor the peak power launched into a $25 \mathrm{~km}$ standard SMF. In the probe branch, an electro-optic modulator (EOM) generates a two-sideband probe with suppressed carrier, and a polarisation switch eliminates the polarisation dependence on the SBS gain. Using a $10 \mathrm{GHz}$ fibre Bragg grating (FBG) and an optical circulator only one probe sideband is selected and then sent to a $125 \mathrm{MHz}$ photo-detector (PD). Finally, a computer acquisition card records the time-domain traces.

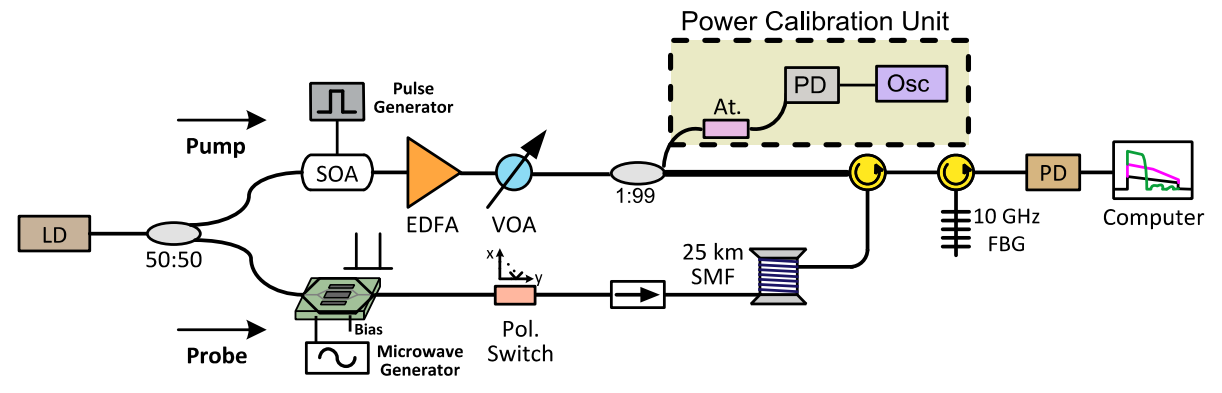

Figure 3. Experimental setup for a $25 \mathrm{~km}$ long BOTDA sensor. LD: laser diode; SOA: semiconductor optical amplifier; EDFA: erbium-doped fibre amplifier; VOA: variable optical attenuator; FBG: fibre Bragg grating; At: $10 \mathrm{~dB}$ attenuator; PD: photodetector; Osc: Oscilloscope; EOM: electric-optic modulator; SMF: single-mode fibre.

Fig. 4 shows the experimental and theoretical results of the depletion length $L_{\mathrm{D}}$ versus the input pump power $P_{0}$ for two values of depletion rate $R_{\mathrm{D}}$, namely 10 and 50 percent. The measurement curves are obtained from the acquired Brillouin traces at the peak gain frequency. The level of depletion is estimated by calculating the power difference between the measured traces and the expected undepleted ones, so that the depletion length $L_{\mathrm{D}}$ (blue lines in Fig. 4) is then obtained at the fibre location where the depletion ratio reaches a given $R_{\mathrm{D}}$ percent. The horizontal top points at $25 \mathrm{~km}$ show that for those values of input power, depletion is less than $R_{\mathrm{D}}$ percent over the $25 \mathrm{~km}$ long fiber. The solid red lines show the depletion length obtained from the Monte Carlo simulation of MI by solving the NLS equation using split-step Fourier transform method. The level of background noise used in the simulation is $S_{n}=-121 \mathrm{dBm} / \mathrm{Hz}$, which is in good agreement with the experiment, in which similar levels of ASE noise are generated by the used EDFA. It is evident from the plots that the measurements and simulations match together perfectly for low pump powers, but differ for high input powers. A possible explanation for this is that, in a high power regime, other nonlinear effects such as SRS can generate additional pump depletion, while the simulations only consider the MI process. Furthermore, the theoretical analysis of the depletion length includes two cases; one is the numerical calculation (green lines in Fig. 4) based on Eq. (2), and the other is the proposed analytical formula (dashed brown lines) given by Eq. (5). It is worth mentioning that the analytical model overestimates the MI effect, so that the corresponding depletion length turns to be slightly shorter than the one predicted by the numerical method. The numerical calculation matches the measurements at low pump power regime better than the analytical model, while for high powers, the analytical model is in perfect agreement with the measurements. Since the numerical solution is more exact than the analytical one, this shows a better agreement with the measurements and simulations for low pump powers, where the only dominant effect is modulation instability. 

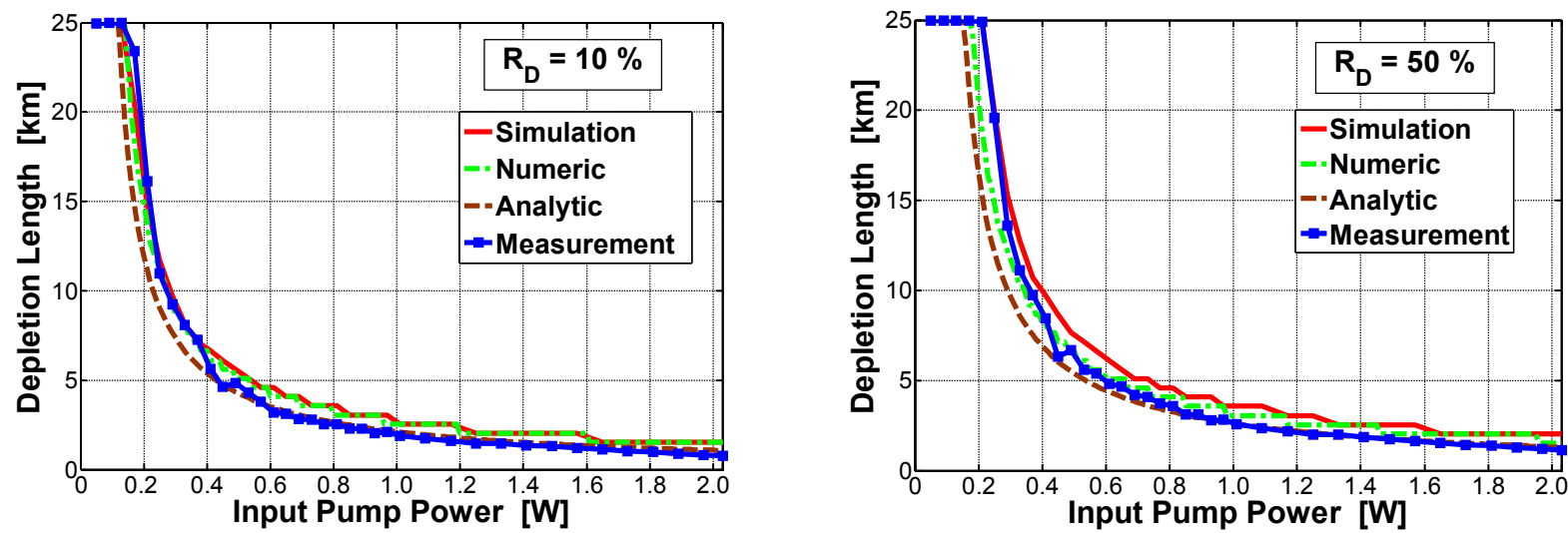

Figure 4. Depletion length $L_{\mathrm{D}}$ vs. input pump power for two values of depletion ratio $R_{\mathrm{D}}$, i.e. 10 and $50 \%$. Figures depict the experimental measurement (blue squares), the Monte Carlo simulation (solid red) and the corresponding theoretical model calculated numerically (dot-dash green) using Eq. (2) and analytically (dash brown) using Eq. (5).

On the other hand, as the tolerable depletion rate increases, the difference between measurements, simulations and analytical results becomes more evident, implying that the model is suitable for low depletion regime; while for high depletion a more sophisticated analysis for the MI gain spectrum considering pump depletion is needed. Since the analytical model is obtained by an overestimated approximation of the MI process, the corresponding depletion length can act as a rule of thumb to guarantee less depletion than the allowable depletion in a distributed optical fibre sensor.

\section{CONCLUSION}

Depletion length and depletion ratio have been introduced to model the effect of pump depletion due to modulation instability in distributed optical fibre sensors. An analytical model for the depletion length has been proposed and its dependence on the background noise level has been clarified. A comparison between measurements, simulations, numerical and analytical solutions has been carried out to validate and evaluate the accuracy of the proposed model. Thanks to the possibility to obtain a closed-form expression the model is a useful tool to design and analyse distributed optical fibre sensors as a function of pump power, fibre length and maximum pump depletion allowed in the system.

\section{ACKNOWLEDGEMENT}

The authors acknowledge the support from the Swiss Commission for Technology and Innovation (Project 13122.1).

\section{REFERENCES}

[1] Foaleng, S. M., and Thévenaz, L., "Impact of Raman scattering and modulation instability on the performances of Brillouin sensors," Proc. of SPIE, vol. 7753, 77539V (2011).

[2] Dong, Y., and Bao, X., "High spatial resolution and long-distance BOTDA using differential Brillouin gain in a dispersion shifted fiber," Proc. SPIE 7503, 750384 (2009).

[3] Alahbabi, M. N., Cho, Y. T., and Newson, T. P., "Influence of modulation instability on distributed optical fiber sensors based on spontaneous Brillouin scattering," J. Opt. Soc. Am. B 21(6), 1156-1160 (2004).

[4] Alasia, D., et al., "Detrimental effect of modulation instability on distributed optical fiber sensors using stimulated Brillouin scattering," Proc. of SPIE, vol. 5855, 587-590 (2005).

[5] Martins, H. F., et al., "Modulation instability-induced visibility fading in phase-sensitive OTDR," Proc. SPIE 8794, 87943E (2013).

[6] Smith, R. G., "Optical power handling capacity of low loss optical fibers as determined by stimulated Raman and Brillouin scattering," App. Opt., 11 (11), 2489 (1972).

[7] Soto, M. A., Alem, M., Chen, W., and Thévenaz, L., "Mitigating modulation instability in Brillouin distributed fibre sensors," Proc. SPIE 8794, 87943J (2013).

[8] Tai, K., Hasegawa, A., and Tomita, A., "Observation of modulation instability in optical fibers," Phys. Rev. Lett., 56 (2), 135 (1986).

[9] Marhic, M. E., Curri, V., and Kazovsky, L. G., "Bessel function solution for the gain of one-pump fiber optical parametric amplifier,” IEEE Proc. Nonlinear Optics 98, TuC21, 221-223 (1998). 